\title{
Smart Collaboration in Global Virtual Teams: The Influence of Culture on Technology Acceptance and Communication Effectiveness
}

\author{
Carolin Fleischmann \\ University of Southern California \\ fleischc@marshall.usc.edu
}

\author{
Peter Cardon \\ University of Southern California \\ cardon@marshall.usc.edu
}

\author{
Jolanta Aritz \\ University of Southern California \\ aritz@marshall.usc.edu
}

\begin{abstract}
Teams are beginning to rely on smart communication technology that is enhanced by Artificial Intelligence (AI). Yet, we lack understanding of how these smart communication technologies (SCT) influence team collaboration, especially in global virtual teams (GVT). This study empirically investigates how cultural values and practices influence the acceptance of SCT and how the use of this technology impacts communication effectiveness in GVT. We surveyed 643 members of 109 GVT before and after using the SCT. Results showed that team members from individualistic, future oriented cultures generally had more positive expectations towards the performance and enjoyment of using the technology. Uncertainty avoidance increased effort expectancy. After using SCT for communicating in the GVT, most differences disappeared. Regarding communication effectiveness, SCT had a positive influence, which was stronger for performance and future oriented cultures.
\end{abstract}

\section{Introduction}

Contemporary organizations rely on teams to solve complex problems. Teams are assembled based on talent rather than close proximity, leading to teams that are nationally diverse, dispersed across countries and time zones, and rely on technology to collaborate. These teams are called global virtual teams (GVT).

Communication technologies are supposed to guarantee seamless collaboration and facilitate the team's ability to complete tasks. Collaboration is tasklevel and social interaction to share resources and knowledge and to jointly accomplish tasks [1]. Communication is at the center of collaboration. While collaboration includes additional aspects, such as coordination, balanced contributions, mutual support and cohesion [2], all forms of collaboration are facilitated by communication. We are therefore focusing our study on communication in GVT.
Which communication technologies are most suitable depends on the goal and context of the communication and the backgrounds of the team members [3] [4] [5].

Recently, these communication technologies have integrated machine learning (ML), natural language processing, and other forms of artificial intelligence (AI), which are often called smart technologies. They use algorithms and data to predict the end of a sentence (e.g. Google Mail); they remind us if we forgot an attachment (e.g. MS Outlook); they judge the appropriateness of our tone (e.g. Grammarly); they transcribe speech-to-text (e.g. Skype); they detect emotions (e.g. Affectiva); and they interpret our facial expressions (e.g. Jargon). By augmenting human communication, smart technologies can to facilitate communication and enhance virtual teamwork.

However, research on whether and how members of diverse teams accept these smart communication technologies (SCTs) remains scarce despite calls for investigating smart technologies in collaborative settings [6]. To investigate the acceptance of smart technology, such as job recommender systems [7], voice assistants [8], and smart meters [9], researchers used the UTAUT2 model.

In a multinational context, several studies have integrated culture into the UTAUT models. Most of these studies have limited their investigation to comparing two countries (e.g., [10]: USA and South Korea; [11]: USA and China) or testing the model for a single cultural context (e.g., [12]: China; [13]: Mozambique).

When looking at smart technologies and team dynamics, some studies have explored trust and reliance in human-machine collaborations (e.g., [14] [15]). Other than these studies, we know little about if and how SCTs alter team interaction.

To learn more, this study focuses on a SCT that we classify as Emotion AI, a subset of AI that detects and interprets human emotions [16]. It assesses verbal (i.e., pronoun use, positivity), non-verbal (i.e., facial expressions, eye contact), and para-verbal (i.e., inflection, pausing) cues to provide insights into the 
meaning of a message beyond words. These SCTs lack scholarly analysis to answer questions about their acceptance and their effect on team communication: whether they decrease misunderstandings and increase civility of team communication, or whether they create anxiety and distrust within the team.

These questions are particularly relevant for multinational teams: Does acceptance of SCTs differ across cultures? Do SCTs bridge the differences and increase communication effectiveness? While team diversity can lead to better results, it also poses risks of miscommunication and misalignment of values [17]. SCTs need to help overcome these barriers rather than reinforce them.

The objective of this study is to investigate (1) whether differences in acceptance of SCT exist across cultures, and (2) how the use of SCTs influences communication effectiveness.

To test our hypotheses we surveyed 643 participants of a virtual team project, who worked in 109 global virtual teams. They completed a quantitative survey questionnaire before the start of the 7-week-long project and at the end of the project.

Our contribution is three-fold. First, we investigate the acceptance of SCT in a novel context GVT - and based on a sparsely researched smart technology - Emotion AI. Second, we expand the knowledge about the influence of culture on technology acceptance in the UTAUT2 model. Third, we offer insights on how SCTs alters teamwork.

\section{Theoretical Background}

\subsection{Communication in GVT}

GVT are teams whose members are located in different countries and rarely meet in person but rely on technology to communicate [18]. We draw on two research streams to understand GVT: Virtual team research, which provides many insights on the dispersion of team members, and Computer-mediated work research, which focuses on the technology aspect of virtual teams [19]. GVT face communication challenges that are less frequently encountered by colocated and nationally homogeneous teams. Most importantly, they are missing many social and emotional cues that are present in face-to-face communication. This leads to difficulties creating trust and accountability in virtual teams. These differences are even more difficult to bridge in a multicultural virtual communication setting because diverse teams have more difficulty to arrive at a shared understanding, i.e. to be on the same page [20]. Their communication is additionally impacted by different native languages, mindsets, and cultures [5]. However, a lack of cues may also make cross-border communication easier because social and emotional cues may be ambiguous and cause mental overload for team members from different cultures [21] [4].

Therefore, communication technologies have to be chosen in a context-dependent way in order to facilitate GVT communication. In media synchronicity theory (MST), communication effectiveness will be highest when the capabilities of the used media match the communication goal [3]. In GVT, team members with lower levels of proficiency in the team's language are less likely to consider synchronous communication effective for negotiating a shared meaning. They benefit from written communication because it allows them to plan their messages more carefully (rehearsability) and reprocess received messages multiple times (reprocessability) [4] [5].

On the other hand, GVT benefit from synchronous communication independent of the communication goal. Team members from teams that hold more online meetings feel more included and satisfied - regardless of their native language and their level of language proficiency [5].

\subsection{Artificial intelligence for global virtual team communication}

SCTs, which are technologies that use AI and ML to detect, understand, and reply to human communication, may be able to integrate the benefits of asynchronous and synchronous communication, e.g. by transcribing (speech-to-text) the contents of an online meeting. Group support systems have been helping teams increase their performance for several decades. Even in the late 1980s, technology channeled information and actions to improve decision-making by filtering information and discouraging unproductive behaviors and interactions [22].

A variety of research suggests AI technologies can improve human collaboration and decision making. Typically, however, this research focuses on how AI technologies can help find, process, and analyze information in a timely manner for teams. The focus of these technologies is to provide "the right information at the right time" [23].

With growing robustness of the predictive quality, SCTs, such as chatbots and other intelligent agents, are increasingly used to ease the communication between humans. They promise to augment virtual communication by facilitating communication, remedying some of the barriers of virtual communication, and providing additional assistance to increase shared understanding.

While different models exist, most SCTs use 
multiple sensors - usually the device's camera and microphone - to capture raw audio-visual cues. The multimodal raw cues are integrated at high frequency and used for a machine learning-based prediction model for human behavior detection. Using an algorithm, 5-10 second chunks of detected behavior are analyzed and serve as the basis for real-time feedback [24]. The average communication behavior is later shown in a report and an annotated transcript of the conversation.

While the most common applications of this technology have focused on communicating with customers, it is increasingly used by colleagues and teams to transcribe meetings, suggest smart replies, and help retrieve information [23] [25]. Some emerging smart technologies, such as the one that the GVT used in our study, Jargon, evaluate team dynamics and provide diagnostic information to the team. This diagnostic approach to team communication is relatively unexplored in research.

In our study, the GVT used Jargon in their video meetings. Jargon is a so-called Passive Pervasive Assistant that does not require explicit user input; the system observes the user's behavior and reacts by providing on-screen feedback [26].

Jargon records every meeting, transcribes it, and analyzes the conversation both real-time and post-hoc. Real-time assessment provides feedback on attitude towards the conversation topic (measured by facial expressions) and engagement (measured by eye contact). After the video meeting, the SCT creates a report with additional analysis of the conversation, such as emotions behind words, rapport, level of profanity in language, facial expressions, turn-taking, number of turns, interruptions, and pace of speech.

With these functionalities, Jargon has the potential to remedy some of the challenges of traditional communication technology: It is a synchronous communication technology, but allows for reprocessability due to the recording and transcript. It allows for rich social and emotional cues but decreases their ambiguity by interpreting them.

However, this type of analysis has to be critically evaluated, particularly in a multicultural context. Communication styles and emotion regulation differ across nations and many other individual traits [21]. However, the algorithms that analyze the verbal and non-verbal messages do not distinguish between cultural backgrounds of the speakers. Decoding meaning with standardized cultural standards and without considering context is a common source of bias. Therefore, careful ethical considerations have to guide the adoption of SCT in GVT [27]. The algorithms need to be trained to recognize and include diverse contexts and users [28].

\subsection{Technology acceptance model for SCT}

Because SCT are controversially discussed, we need to investigate their acceptance among different user groups. We base our research on the UTAUT models [29] [30], which are among the most prominent and widely used technology acceptance models. In UTAUT, intention to use a technology is influenced by several factors, such as performance expectancy, effort expectancy and attitude (UTAUT)/ hedonic motivation (UTAUT2). The intention to use a technology, in turn, influences the actual use.

In UTAUT2, performance expectancy, effort expectancy, social influence, facilitating conditions, hedonic motivation, price value, and habit influence behavior intention [30]. These factors need to be adjusted to account for the set-up of our study. We excluded social influence because of the involuntariness of using the technology; facilitating conditions because every participant in the study had the same resources and support to use the technology; price value because the SCT was free of charge for study participants; and habit because the participants have not been exposed to the SCT before. UTAUT2 was specifically developed to measure acceptance of consumer technology [30]. Therefore, we also drew on the original UTAUT model to account for the organizational context of our study.

We focused on individuals' performance expectancy (PE), effort expectancy (EFF), and hedonic motivation (HED) and measured these factors before participants used the SCT for the first time. Past studies found that these variables have been a significant predictor for the intention to use a technology [8] [7] [13]. PE, in particular, has a strong influence on behavior intention [29] [30]. PE and EFF represent extrinsic motivation. PE measures whether individuals expect a technology to perform and support performance or whether the technology rather detracts from the actual task and therefore hinders performance [1]. While PE is an outcome-oriented factor, EFF measures the ease of use of the technology and whether users believe that they can independently and easily navigate the technology. HED represents the attitudes towards the process of using the technology and measures intrinsic motivation. When it is fun to use a technology, people have a higher intention to use it [31] [8].

\subsection{Cultural values and practices: the GLOBE study}

Individuals from different cultures may accept new technologies to different extents, depending on their cultural background. The GLOBE study of 
culture is an extension and refinement of Hofstede's cultural dimensions. While Hofstede focused on workrelated values, GLOBE distinguishes between practices and values, which are sometimes negatively correlated. Practices reflect how values are implemented in a society; values reflect people's perception of how values should be implemented in a society [32]. This distinction is particularly important in a study that measures both attitudes (pre-use) towards as well as experiences (post-use) with a technology. Therefore, we use GLOBE values to predict pre-use expectations and GLOBE practices to explain post-use experiences.

The GLOBE study identifies eight cultural dimensions: Uncertainty Avoidance (UAV), Future Orientation (FO), Humane Orientation (HUM), Individualism and Collectivism (COLL), Performance Orientation (PO), Gender Egalitarianism (GE), Assertiveness (ASS), and Power Distance (PD) [32]. Only a subset of these are relevant for technology acceptance.

GLOBE discussed technology in the context of three dimensions: UAV, FO, and COLL. UAV represents the degree to which someone desires structure and consistency to increase predictability of future events. FO stands for planning, investing in the future, and the willingness to delay rewards. COLL measures the degree to which individuals value cohesive groups and loyalty towards the in-group [32].

In addition to these, we also include HUM and PO as predictors of technology acceptance. HUM measures the degree to which someone values caring and kind interpersonal relationships, which may be influenced by SCT. PO measures the commitment towards performance and excellence, which seems relevant in a context where SCT is supposed to foster team performance [32].

As outlined in Figure 1, we model these cultural dimensions as predictors rather than moderators of technology expectation and experience. Our approach is in line with past research, which has shown that cultural dimensions are independent predictors (e.g., [33] [11]). At the same time, we acknowledge that other studies have found a significant moderating influence of cultural dimensions on technology acceptance (e.g., [13]).

According to GLOBE, UAV is most strongly correlated with technology advancements in a society. Societies that are high in UAV value formalization and structure, which in turn lead to more efficient technology transfer and more focus on research and technology as a means to reduce uncertainty. UAV cultures seek structure and order which may be supported by SCT. Individuals with high UAV also reflect more on the effort of using the new technology [33]. While high UAV cultures are likely to resist change more often, that resistance does not seem to apply to technology [32]. Studies that have used Hofstede and GLOBE dimension to investigate technology acceptance across cultures, have often hypothesized and found the contrary relationship: in order to avoid uncertainty, high UAV cultures may resist or delay the adoption of new technologies (e.g. [34]). This effect may be explained by higher levels of anxiety and thus low levels of enjoyment related to change in high UAV cultures. To combine both perspectives, we hypothesize:

H1: With increasing UAV, (a) PE and (b) EFF will increase and (c) HED will decrease.

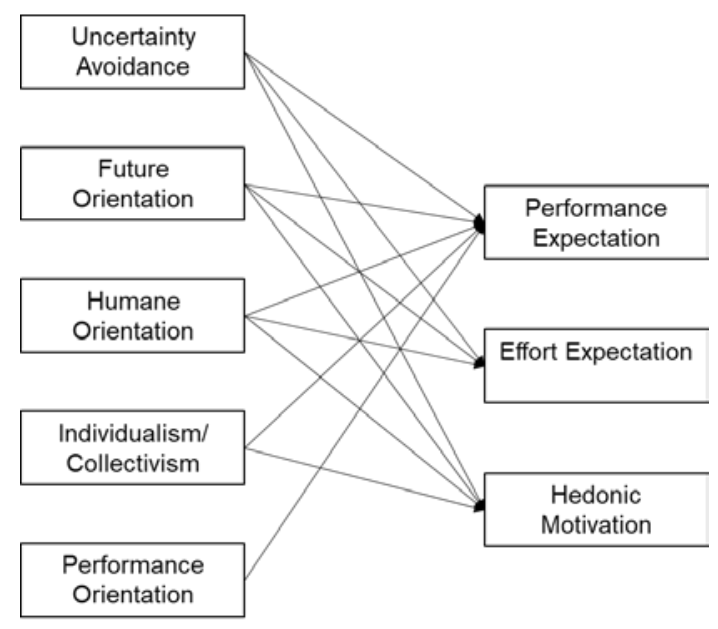

\section{Figure 1. Modelled impact of GLOBE cultural dimensions on the antecedents of technology acceptance}

In the GLOBE study, success in science and technology is positively correlated with FO practices. Individuals with high FO are willing to adapt to changing circumstances [35], such as a new technology, and invest effort into making it work. FO therefore leads to higher technology performance and a higher effort. For future success, hedonic motivation may be sacrificed in high FO societies, while low FO is related to hedonic pleasure [32]. We hypothesize:

H2: With increasing FO, (a) PE and (b) EFF will increase and (c) HED will decrease.

HUM is characterized by interpersonal support and informal relationships. The use of SCT may cause fear of a formalized technological instead of humancentered way of relationship development. They may also have ethical concerns regarding privacy, machine-guided understanding of emotions, and the correctness of the technology's analysis. Team members with high HUM will not anticipate value 
from using the technology and see it as a burden and threat rather than a facilitator. Therefore, we hypothesize:

H3: With increasing HUM, (a) PE will decrease, (b) EFF will increase, and (c) HED will decrease.

In the GLOBE study, in-group COLL values and practices have a negative impact on a society's success in basic science and technology transfer. Social structures and relationships are more important than technology or performance. Because collectivists highly value human relationship building, we expect skepticism towards a machine agent that influences team interaction. Computerized behavior detection may threaten the collectivists' desire for a controlled self-presentation. EFF is not expected to be influenced by COLL. We therefore hypothesize:

H4: With increasing COLL, (a) PE and (b) HED will decrease.

Lastly, high PO represents societies that emphasize results and individuals' control over outcomes. Individuals have their fate in their own hands. They prioritize results over people and value feedback as a necessity for improvement [32]. The SCT, Jargon, satisfies these preferences. We do not expect PO to influence EFF and HED. Therefore, we hypothesize:

H5: With increasing PO, PE will increase.

We hypothesize similar relationships between cultural dimensions and technology acceptance preand post-project. In only one case, we hypothesize a contrary relationship pre- and post-use: The influence of UAV on HED. While high UAV individuals are expected to be too anxious about the new technology to expect it to be fun and interesting, we expect them to enjoy using the technology once they know how it works, i.e. after they have used it in the project. The technology will reduce their uncertainty about communicating with their teammates. Therefore, for post-project analysis, H1c changes to:

H1c(post): With increasing UAV, HED will increase.

The second objective of our study is to investigate whether and how the use of SCT influences communication effectiveness in a GVT. The SCT is supposed to augment virtual communication by alleviating usual barriers of virtual communication (e.g. lack of social and emotional cues) while providing additional assistance to increase shared understanding (e.g. adding reprocessability by providing a transcript). Therefore, we expect:

H6: The use of SCT will increase communication effectiveness in a GVT.

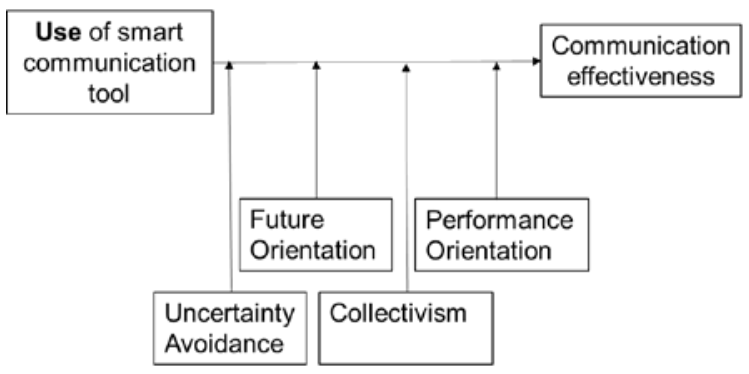

Figure 2. Communication effectiveness in GVT using SCT

Culture may moderate this positive relationship between the use of the SCT and communication effectiveness (Figure 2). Our rationale for these relationships is grounded in our hypotheses 1-5. The SCT, which provides a report on metrics of a meeting, will be useful information for a person with high UAV. Individuals with high UAV will use the information in the report to satisfy their need to decrease uncertainty. Individuals with high UAV will therefore perceive the team's communication as more effective when using the SCT. Team members high in FO will also perceive communication effectiveness to go up with increasing use of the SCT. The report and transcript that the technology provides would satisfy their desire to plan. Team members with high PO will also perceive the communication effectiveness to increase with SCT because they view feedback as necessary for success [32]. COLL will negatively influence the relationship between the use of SCT and communication effectiveness. While individualists may use insights from the report to their advantage, collectivists may feel that an individual level analysis is impeding the collective unit of the team and is therefore detrimental to communication effectiveness.

H7: Culture moderates the effect of SCT use on communication effectiveness, such that the effect is stronger for cultures with higher (a) UAV, (b) FO, (c) PO and lower (d) COLL.

\section{Methodology}

\subsection{Sample}

The participants in our study worked in virtual teams and used SCT, Jargon, to hold video meetings 
with teammates dispersed around the globe. The 7week project required participants to collaborate on a consulting project for one of three US-based Fortune 100 companies. The final deliverable was a written report that included analysis and recommendations.

In the project, undergraduate and MBA students from various disciplines were placed in GVT that included members from different institutions and countries. They never met in person. The data was collected in February and April 2019 from project participants from over 14 universities in nine countries, including - by number of participants - the United States, India, Canada, Lithuania, Finland, Spain, France, Germany, and Singapore.

A total of 643 students in 109 teams participated in the project. Students worked in teams of 5 or 6 members of similar diversity. Due to the high number of US-based students in the sample, every team had two to three US-based team members from different institutions and time zones, one Indian team member, and two to three team members from other countries. Every team represented students from six different institutions in at least three different countries.

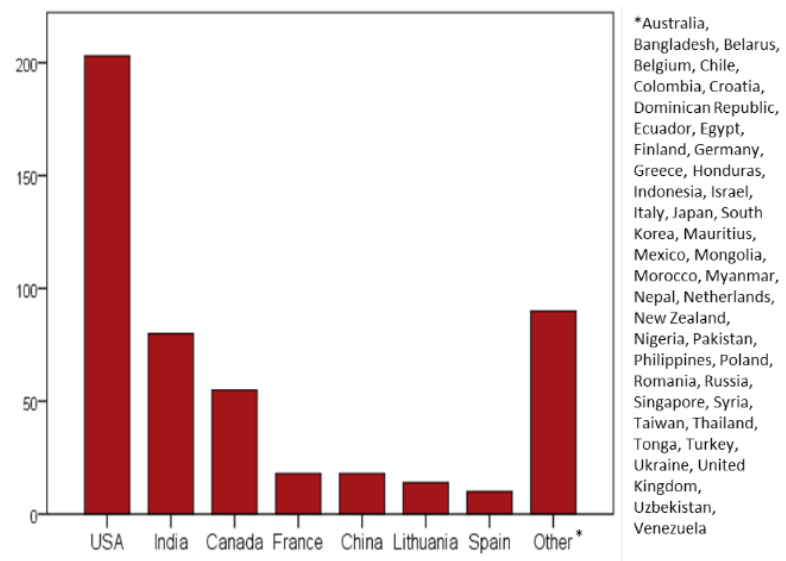

Figure 3: Nationalities of survey participants

Participants were asked to complete two quantitative surveys - one at the beginning and one at the end of the project. The surveys included identifiers to match the responses of pre- and post-survey. They were available in English language to ensure semantic equivalence. All study participants had a working level of English proficiency, which was assessed by formal test scores, professors' ratings, and self-ratings. 544 participants completed the pre-project survey (44.2\% male; $55.2 \%$ female, $0.2 \%$ other); 546 participants completed the post-project survey (response rate $85 \%$ ). While the participants studied in one of nine countries, the survey respondents originally came from 50 different countries. Their nationalities and absolute numbers of respondents per nationality are indicated in Figure 3.

\subsection{Measures}

For our analyses, we used measures to assess (a) expectations about SCT (pre-project), (b) experiences with and use of SCT (post-project), (c) cultural values and practices, and (d) communication effectiveness.

To measure expectations and experiences with SCT, we used the well-established measures from Venkatesh et al.'s UTAUT models. Due to the specific context of our study, we adapted the model to fit our study design. As outlined in 2.3, we limited our analysis to PE, EFF, and HED. All three constructs were measured pre- and post-project. The 4-itemmeasure of PE was adopted from the original UTAUT model [29] to include an item on the organizational and project context (pre: "The AI communication tool will increase my team's chances of getting a better grade”; post: “Jargon increased my team's chances of getting a better grade.”). EFF and HED were adopted from UTAUT2 [30]. EFF has four items, e.g. "I think the AI communication tool will be easy to use" (presurvey) and "Jargon was easy to use" (post-survey). HED included three items, e.g. "Using the AI communication tool will be fun” (pre-survey) and "Using Jargon was fun” (post-survey). All items were measured on a 7-point Likert scale from $1=$ completely disagree to $7=$ completely agree. In line with the UTAUT2 measure, use of SCT was assessed by frequency of use of different features of the SCT. Namely, survey participants indicated on a scale from $1=$ never to 7 = for all team meetings how frequently they used the video conferencing tool, the audio recording, the transcript, and the report.

GLOBE scores (on a 7-point scale) for cultural values and practices were assigned according to participants' nationalities [32]. Some nationalities in our sample were not represented in the GLOBE study (e.g., Belgium, Mongolia, Uzbekistan). Therefore, these individuals had to be excluded from the analysis. The final sample size that we used for the analyses was $n=435$. We used cultural values to predict expectations towards SCT (pre) and cultural practices to predict experiences with SCT (post). The rationale behind this decision is that values, as an assessment of what should be, reflect what survey participants believe and wish for. They are therefore relevant in hypothetical situations, such as an assessment of a technology that an individual has not yet used. Practices are an assessment of what is. These actual behaviors surface when the participants use the SCT and assess their experiences with it.

Communication effectiveness was measured by integrating two scales from Early and Mosakowski 
[36]. Internal communication is a 2-item scale (e.g., „We do not seem to understand what one another is saying during our discussions.“ (reverse coded). Team communication is a 4-item scale (e.g., , We freely express our feelings and ideas.”). The items were measured on a 7-point Likert scale from $1=$ completely disagree to $7=$ completely agree .

\section{Results}

Before testing the exact effects of the GLOBE cultural dimensions on the antecedents of technology acceptance, we assessed whether PE, EFF, and HED even differed across nationalities. All three constructs show significant differences across cultures (PE: $\mathrm{F}=$ 1.975, $\mathrm{p}<0.001$; EFF: $\mathrm{F}=1.907, \mathrm{p}<0.001$; HED: $\mathrm{F}$ $=2.387, \mathrm{p}<0.001)$.

To test hypotheses H1-5, we conducted multivariate OLS regression analyses (table 1 and 2).

Table 1. Cultural values' influence on technology expectations (pre-use)

\begin{tabular}{llll}
\hline & PE & EFF & HED \\
\hline UAV & -.046 & $.177^{*}$ & -.022 \\
FO & $.192^{* *}$ & .073 & $.235^{* *}$ \\
HUM & -.082 & -.063 & -.014 \\
COLL & $-.227^{* *}$ & & $-.306^{* *}$ \\
PO & .035 & &
\end{tabular}

standardized $\beta$ coefficients are reported

${ }^{* *} \mathrm{p}<0.001,{ }^{*} \mathrm{p}<0.005$

Before having used the SCT, PE was positively influenced by FO $(\beta=0.192, \mathrm{p}<0.001)$ and negatively influenced by COLL $(\beta=-0.227, \mathrm{p}<0.001)$. EFF was only influenced by UAV $(\beta=0.177, \mathrm{p}<0.005)$ but no other tested cultural value. FO significantly and positively impacted HED $(\beta=0.235, \mathrm{p}<0.001)$, while COLL negatively influenced HED $(\beta=-0.306, \mathrm{p}<$ 0.001). HUM and PO did not have a significant effect on any factor of technology acceptance.

\section{Table 2. Cultural practices' influence on technology experiences (post-use)}

\begin{tabular}{lccc}
\hline & PE & EFF & HED \\
\hline UAV & -.094 & .027 & -.059 \\
FO & -.046 & -.085 & .060 \\
HUM & -.038 & .028 & -.071 \\
COLL & $.152^{*}$ & & $.233^{* *}$ \\
PO & .083 & & \\
\hline
\end{tabular}

standardized $\beta$ coefficients are reported

${ }^{* *} \mathrm{p}<0.005,{ }^{*} \mathrm{p}<0.05$

To test H6 and H7, we ran a set of five OLS regressions, where we included the hypothesized GLOBE cultural dimensions as moderators.
Generally, the use of the SCT increased communication performance $(\beta=0.216, \mathrm{p}<0.005$; model 1 in table 3 ). As indicated in table 3, FO (model 3) and PO (model 5) partly moderated the relationship between use of the technology and communication performance. Including these cultural dimensions improved the model significantly (see $\Delta R^{2}$ and $\Delta F$ ). While model 4 yields a better fit than model 1 , this effect is due to COLL as an independent variable rather than a moderating effect.

Table 3. Impact of technology use and culture on communication effectiveness

\begin{tabular}{llccc}
\hline Model & $\beta$ & $\Delta R^{2}$ & $\Delta F$ \\
\hline 1 & Technology Use & $.130^{* *}$ & $.130\left(R^{2}\right)$ & $3.406^{*}(F)$ \\
\hline 2 & Technology Use & $.106^{*}$ & -.012 & .302 \\
& UAV & -.033 & & \\
& Tech Use x UAV & .032 & & \\
\hline 3 & Technology Use & $.108^{*}$ & .033 & $2.516^{*}$ \\
& FO & -.084 & & \\
& Tech Use x FO & $.108^{*}$ & & \\
\hline 4 & Technology Use & .082 & .056 & $3.833^{*}$ \\
& COLL & $.153^{* *}$ & & \\
& Tech Use x COLL & .013 & & \\
\hline 5 & Technology Use & $.102^{*}$ & .037 & $3.001^{*}$ \\
& PO & $-.123^{*}$ & & \\
& Tech Use x PO & $.102^{*}$ & & \\
${ }^{* * *} \mathrm{p}<0.005,{ }^{*} \mathrm{p}<0.05$ & & & \\
&
\end{tabular}

In summary, our data supports H1b, H2a, and H4 (all three pre-project), H6, H7b, and H7c. For H2c (pre-project) and $\mathrm{H} 4$ (post-project), data shows the opposite relationship. The other hypotheses are not supported.

\section{Discussion}

For a GVT context, this study revealed that uncertainty avoidance, future orientation, and collectivism influence the expectations and experiences with a SCT that detects and analyzes communication behavior. Furthermore, the use of the SCT influences communication effectiveness in a GVT. Future orientation and performance orientation moderate the relationship between the use of the SCT and communication effectiveness.

This research contributes to the body of knowledge on the acceptance of smart technology by presenting empirical evidence on the acceptance of SCT in a GVT context. We apply parts of the UTAUT2 model to a team setting, thus expanding the model's scope and deepening the insights into culturedependent technology acceptance. Hence, we provide evidence that cultural values and practices are antecedents of smart technology acceptance and user 
motivation. Lastly, we contribute to the understanding of how smart technology impacts communication in a GVT. We specifically evaluate an Emotion AI solution, Jargon, for its contribution to overcoming cross-cultural communication barriers. As such, we are adding to theory development by showing that team members use Emotion AI's advanced communication abilities to improve their teamwork.

For understanding technology acceptance, we assessed three dimensions - performance expectancy, effort expectancy, and hedonic motivation - both before teams worked with SCT and after the global virtual team project had finished. While we observed several differences in technology acceptance across cultures before the start of the project, most of these differences disappeared post-project.

Before the project began, team members from countries with high future orientation and individualism expected SCT to perform well. Future oriented cultures focus on the long-term outcome rather than short-term considerations. Therefore, they trust in long-term technological advancement and the positive effects of technology on performance. Along similar lines, individualistic cultures believe in technology's capabilities to augment human cognition and ultimately performance, whereas collectivist cultures may be concerned that a technology that detects behaviors and emotion would impact the relationships with their teammates. They fear losing the opportunity to choose a certain way of presenting themselves to their team members [32].

Other than performance expectancy, effort expectancy was influenced by culture. Individuals from cultures with high uncertainty avoidance expected the effort of using SCT to be high. Putting a lot of effort into understanding the technology and building the necessary skills to work with it reduces uncertainty with an unfamiliar technology and is therefore something that team members from uncertainty avoiding cultures would do.

In the context of hedonic motivation, future orientation and individualism are - once again predictors. For similar reasons as outlined above, individualists are intrinsically motivated to use SCT. Without worrying about the effect on the team's collaboration, they value the new experience. While one could expect individualists to be concerned about privacy or emotion detection by a machine, this effect is not represented in our data. Future orientation also impacted hedonic motivation. Contrary to our hypothesis, team members from countries with high future orientation had high hedonic motivation. Their hedonic motivation may be a spill-over effect from performance orientation such that they believe in the future performance of the technology so much that they become intrinsically motivated and enjoy using the SCT in the present.

After the GVT had worked with the SCT for seven weeks, most cultural differences in perception of the technology had vanished. Team dynamics and team climate influence how a team uses a technology and, hence, how individual team members experience the SCT. These team effects seem to dominate the acceptance of the technology post-project [37].

Only collectivist orientation still had a significant influence on performance and hedonic motivation. However, that influence was reverse from what we observed pre-project: Higher collectivism was now associated with performance and hedonic motivation. Despite being skeptical before the project started, team members from collectivist countries learned to appreciate SCT for their team's needs. By analyzing the team's communication, the SCT had very teamand interaction-oriented characteristics. Also, providing a transcript and recording of the meeting is a way to include all team members, including those who were absent during a meeting. These features seem to have resonated with team members from collectivist cultures.

While the first part of this study focused on the direct individual perceptions of SCT, the second part investigated how smart communication technology influenced communication effectiveness in GVT. The use of SCT had a direct positive influence on communication effectiveness, so the SCT delivered on its promise. It augmented the understanding between team members by detecting and understanding social and emotional cues in addition to verbal cues. Therefore, it was able to decrease misunderstandings in GVT. In addition, the transcripts gave team members a searchable record of the meeting that they could use for future reference.

That relationship was partly moderated by future orientation and performance orientation. For team members from cultures with high future orientation and high performance orientation, the effect of technology use on communication effectiveness was higher. These results are in line with the literature on virtual team communication. Virtual teams need more time than collocated teams to get up to speed and arrive at decisions [38]. Team members who are longterm oriented are more likely to perceive SCT to increase communication effectiveness because they have the long-term goal in mind and are willing to accept that it takes time to learn the technology and build effective communication in a GVT. Likewise, performance orientation is a mindset that allows individuals to use technological advancements for more effective communication and ultimately performance. Individuals from countries with high PO believe that feedback on the team's communication increases communication effectiveness [32]. 
Difficulties with technology use or functioning do not discourage future oriented and performance oriented team members.

\section{Limitations and directions for further research}

Despite its contributions to understanding acceptance and impact of a relatively new technology, the present study has some limitations. These may serve as starting points for further research in the field.

One of the largest limitations is that we deduced the values of all cultural dimensions from participants' nationalities. However, cultures are not homogeneous. Instead, members of a culture differ in their values and practices. Cultural dimensions are a macro-level perspective that neglects micro-level difference between members of a culture. In order to gain more accurate insights, the GLOBE questionnaire would need to be included in the survey study.

Additionally, future research should further investigate the effects of team dynamics on the acceptance of SCT. Our results indicate that team effects level out cultural differences in technology acceptance. In a next step, we need to investigate how exactly team dynamics influence technology acceptance and how SCT influence communication and more generally interaction in GVT.

Lastly, trust in SCT should be included in future studies. Trust is discussed in the GVT literature [39] and in the smart technology, particularly Emotion AI, literature [14] [7] [15]. We therefore expect that trust plays a role for acceptance of SCT and for team communication when using the technology.

\section{Conclusion}

This paper investigated whether differences in acceptance of SCTs exist across cultures, and how team dynamics are influenced by the use of SCT. Specifically, we researched whether certain antecedents of SCT acceptance, as adapted from the UTAUT models, are influenced by cultural values and practices, both before and after using the technology. In a second step, we assessed communication effectiveness after the teams had used the SCT.

Our data showed that culture is a predicator for the expectations about SCT before team used the technology. As such, SCT may create yet another difference between cultures instead of bridging barriers. However, after having used the SCT for seven weeks, team dynamics overlaid the individual, culturally-influenced perceptions of the SCT. The experiences with the technology were similar across cultures. Collectivism was the only dimension that played a role for post-use perceptions. We therefore conclude that SCTs do indeed help to overcome communication barriers in GVT.

The SCT, which is a Passive Pervasive Agent [26] and characterized as Emotion AI [27], leads to higher communication effectiveness in GVT. This effect is more pronounced for future oriented and performance oriented team members. They seem to focus more on the positive effects of enhancing understanding and reprocessability of verbal communication, rather than being concerned about technology failure, ethical concerns or difficulties using the technology. Thus, managers should implement Emotion AI for relationship building and task accomplishment particularly when team members are high in future and performance orientation.

Before embedding smart communication technology in a global virtual team's communication infrastructure, managers should particularly educate team members from cultures with high uncertainty avoidance, collectivism, and low future orientation about benefits of the technology, such as improved mutual understanding. AI-based technologies cause skepticism, particularly in some cultural environments. Ethical considerations have to guide the adoption of Emotion AI in group settings. For example, systems should recognize and honor contextand culture-specific forms of communicating and displaying emotions. Once initial expectations about SCT become more positive across cultures, the overall positive effect of SCT on communication effectiveness may be enhanced even further.

\section{References}

[1] I. Boughzala, G.-J. de Vreede and M. Limayem, "Team Collaboration in Virtual Worlds: Editorial to the Special Issue," JAIS, vol. 13, pp. 714-734, 2012.

[2] M. Hoegl, K. Weinkauf and H. G. Gemuenden, "Interteam Coordination, Project Commitment, and Teamwork in Multiteam R\&D Projects: A Longitudinal Study," Org. Sc., vol. 15, no. 1, p. 38-55, 2004.

[3] A. R. Dennis, R. M. Fuller and J. S. Valacich, "Media, tasks, and communication processes: A theory of media synchronicity," MISQ, vol. 32, no. 3, pp. 575-600, 2008.

[4] H. Tenzer and M. Pudelko, "Media choice in multilingual virtual teams," J. of Int. Bus. Studies, vol. 47, no. 4, pp. 427-452, 2016.

[5] C. Fleischmann, J. Aritz and P. Cardon, "Language Proficiency and Media Synchronicity Theory: The Impact of Media Capabilities on Satisfaction and Inclusion in Multilingual Virtual Teams," in Proc. of HICSS 52, Maui, HI, 2019.

[6] I. Seeber, E. Bittner, R. O. Briggs, G.-J. de Vreede, T. de Vreede, D. Druckenmiller, R. Maier, A. B. Merz, S. 
Oeste-Reiß, N. Randrup, G. Schwabe and M. Söllner, "Machines as Teammates: A Collaboration Research Agenda," in Proc. of HICSS 51, Big Island, HI, 2018.

[7] S. Laumer, F. Gubler, C. Maier and T. Weitzel, "Job Seekers' Acceptance of Job Recommender Systems: Results of an Empirical Study," in Proc. HICSS 51, Big Island, HI, 2018.

[8] K. Wagner, F. Nimmermann and H. Schramm-Klein, "Is It Human? The Role of Anthropomorphism as a Driver for the Successful Acceptance of Digital Voice Assistants," in Proc. of HICSS 52, Maui, HI, 2019. [9] P. Wunderlich, D. Veit and S. Sarker, "Adoption of Sustainable Technology: A Mixed-Methods Study of German Households," MISQ, vol. 43, no. 2, pp. 673-691, 2019.

[10] I. Im, S. Hong and M. S. Kang, "An international Comparison of Technology Adoption Testing the UTAUT model," Information \& Mgt., vol. 48, no. 1, pp. 1-8, 2011. [11] J. Lu, C. S. Yu, C. Liu and J. Wei, "Comparison of mobile shopping continuance intention between China and USA from an espoused cultural perspective," Comp. in Hum. Behavior, vol. 75, pp. 130-146, 2017.

[12] V. Venkatesh and X. J. Zhang, "Unified Theory of Acceptance and Use of Technology: US Vs. China," J. of Global Inf. Tech. Mgt., vol. 13, no. 1, pp. 5-27, 2010.

[13] G. Baptista and T. Oliveira, "Understanding mobile banking: The unified theory of acceptance and use of technology combined with cultural moderators," Comp. in Hum. Behavior, vol. 50, no. Sep, pp. 418-430, 2015.

[14] J. Elson, D. Derrick and G. Ligon, "Examining Trust and Reliance in Collaborations between Humans and Automated Agents," in Proc. HICSS 51, Big Island, HI, 2018.

[15] N. McNeese, D. Demir, E. Chiou and G. Yanikian, "Understanding the Role of Trust in Human-Autonomy Teaming,", in Proc. HICSS 52, Maui, HI, 2019.

[16] M. Somers, "Emotion AI, explained," MIT Sloan School of Mgt., 8.3.2019. https://mitsloan.mit.edu/ ideasmade-to-matter/emotion-ai-explained. [Accessed 7.6.2019].

[17] C. Fleischmann, L. -C. Folter and J. Aritz, "The impact of perceived foreign language proficiency on hybrid team culture," Int. J. of Bus. Comm., 2017 (in press).

[18] M. O'Leary and J. Cummings, "The Spatial, Temporal, and Configurational Characteristics of Geographic

Dispersion in Teams," MISQ, vol. 31, no. 3, pp. 433-452, 2007.

[19] S. Raghuram, S. Hill, J. Gibbs and L. Maruping, "Virtual Work: Bridging Research Clusters", Ac. of Mgt. Annals, vol. 13, no. 1, pp. 308-341, 2019.

[20] D. Fisher, S. Bell, E. Dierdorff and J. Belohlav, "Facet Personality and Surface-Level Diversity as Team Mental Model Antecedents: Implications for Implicit Coordination," J. of App. Psych., vol. 97, no. 4, p. 825-841, 2012.

[21] J. F. George, M. Gupta, G. Giordano, A. M. Mills, V. M. Tennant and C. C. Lewis, "The Effects of Communication Media and Culture on Deception Detection Accuracy," MISQ, vol. 42, no. 2, pp. 551-575, 2018.
[22] J. F. Nunamaker, A. R. Dennis, J. Valacich, D. R. Vogel and J. F. George, "Electronic Meeting Systems to Support Group Work," Comm. ACM, vol. 34, no. 7, pp. 4061, 1991.

[23] H. J. Wilson and P. R. Daugherty, "Collaborative Intelligence: Humans and AI Are Joining Forces," HBR, vol. 96, no. 4, pp. 114-123, July-August 2018.

[24] M. Chollet, P. Ghate and S. Scherer, "A Generic Platform for Training Social Skills with Adaptative Virtual Agents," in Proc. of the 17th Int. Conf. on Autonomous Agents and Multiagent Sys., Stockholm, Sweden, 2018.

[25] J. Kim and J. A. Shah, "Improving Teams' Consistency of Understanding in Meetings," IEEE Transac. on Hum.-Machine Sys., vol. 46, no. 5, pp. 625637, 2016.

[26] R. Knote, A. Janson, M. Söllner and J. M. Leinmeister, "Classifying Smart Personal Assistants: An Empirical Cluster Analysis," Proc. HICSS 52, Maui, 2019. [27] S. Kleber, "3 Ways AI Is Getting More Emotional," HBR, 31 July 2018.

[28] V. Venkatesh, M. Morris, G. Davis and F. Davis, "User acceptance of information technology: Toward a unified view," MISQ, vol. 27, no. 3, pp. 425-478, 2003. [29] V. Venkatesh, J. Y. Thong and X. Xu, "Consumer Acceptance and Use of Information Technology: Extengin the Unified Theory of Acceptance and Use of Technology," MISQ, vol. 36, no. 1, pp. 157-178, 2012.

[30] F. Davis, R. Bagozzi, and P. Warshaw, "Extrinsic and intrinsic motivation to use computers in the workplace," $J$. of App. Soc. Psych., vol. 22, no. 14, pp. 1111-1132, 1992.

[31] R. J. House, P. J. Hanges, M. Javidan, P. W. Dorfman and V. Gupta, Culture, leadership, and organizations: The GLOBE study of 62 societies, Thousand Oaks: Sage, 2004. [32] N. Nistor, T. Lerche, A. Weinberger, C. Ceobanu and O. Heymann, "Towards the integration of culture into the Unified Theory of Acceptance and Use of Technology," British J. of Ed. Tech., vol. 45, no. 1, pp. 36-55, 2014.

[33] D. Leidner and T. Kayworth, "A Review of Culture in Information Systems Research: Toward a Theory of Information Technology Culture Conflict," MISQ, vol. 30, no. 2, pp. 357-399, 2006.

[34] G. Hofstede, "Dimensionalizing Cultures: The Hofstede Model in Context.," Online Readings in Psych. and Culture, vol. 2, no. 1, 2011.

[35] P. Earley and E. Mosakowski, "Creating Hybrid Team Cultures: An Empirical Test of Transnational Team Functioning," Ac. of Mgt. J., vol. 43, no. 1, pp. 26-49, 2000.

[36] H. Liang, Y. Xue, W. Ke and K. K. Wei, "Understanding the Influence of Team Climate on IT USE," JAIS., vol. 11, no. 8, pp. 414-432, 2010.

[37] J. Pridmore and G. Phillips-Wren, "Assessing decision making quality in face-to-face teams versus virtual teams in a virtual world," J. of Dec. Sys., vol. 20, pp. 283-308, 2011.

[38] L. P. Robert and S. You, "Are you satisfied yet? Shared leadership, individual trust, autonomy, and satisfaction in virtual teams," J. of the Assoc. of Inf. Sc. and Tech., vol. 69, no. 4, pp. 503-513, 2018. 
Page 373 